\title{
Regulation of Cyclic AMP-Response Element Binding Protein Zhangfei (CREBZF) Expression by Estrogen in Mouse Uterus
}

\author{
${ }^{\dagger}$ Hoon Jang \\ Dept. of Biomedical Science, College of Life Sciences, CHA University, Seongnam 13488, Korea
}

\begin{abstract}
CREBZF (cAMP-response element binding protein zhangfei) is a member of ATF/CREB family, and which regulates various cellular functions by suppressing major factors with direct interaction. In this study, we have examined the expression of CREBZF on mouse endometrium during uterus estrous cycles and estrogen (E2) treatment. In uterus, CREBZF mRNA expression was higher than other organs and mRNA and protein of CREBZF was increased in proestrus phase and decreased in estrus phase. The expression of CREBZF in 3-weeks old mouse uterus was reduced by E2 injection in endometrium. In addition, the expression of progesterone receptor, a marker of E2 in ovariectomized mice was found to be strongly expressed in stroma, while CREBZF was only expressed in epithelium. Also, we conformed that E2-suppressed CREBZF was restored by co-injection of ICI 182,780, an estrogen receptor antagonist. Overall, these results suggest that CREBZF is regulated by estrogen and involved in ER signaling pathway in mouse uterus.
\end{abstract}

Key words : Cyclic AMP-response element binding protein zhangfei (CREBZF), Endometrium, Estrogen, Uterus

\section{INTRODUCTION}

The uterus is essential organ for reproduction of most mammals, and regulated by several specific steroid hormones as estrogen (E2) and progesterone (P4) in endometrium during uterus cycles (Martín et al., 2002; Riesewijk et al., 2003). Endometrium, one of the three layers of the uterus, is the most active layer and composed with luminal epithelium (LE), glandular epithelium (GE), and stroma (Hu et al., 2004; Huang et al., 2012). Repeated implantation failure (RIF) is occurred due to dysfunction of uterine receptivity with abnormal gene expression in the endometrium. Recent studies attempt to fine the cause of fertilization failure, but it remains as yet unknown due to lack of research on the signaling pathway.

The E2 plays important roles in physiological regulations in the whole body including reproductive organ, central nerve system, skeletal tissues and vascular (Gruber et al., 2002; Hewitt et al., 2005). E2 activities are mediated by the estrogen receptor alpha $(\mathrm{ER} \alpha)$ and estrogen receptor beta $(\mathrm{ER} \beta)$. E2 and ERs are up regulated by insulin resistance, and insulin resistance-mediated inflammation might be modulator for estrogen activity (Calle \& Kaaks, 2004). cyclic AMP-response element binding protein zhangfei (CREBZF) is an insulin-inducible co-repressor during gluconeogenesis in hepatocyte, and insulin resistance decreases CREBZF expression (Lee et al., 2016).

CREBZF belongs to basic region of leucine zipper (bZIP)

Manuscript received February 22, 2018, Received in revised form March 11, 2018, Accepted March 24, 2018

${ }^{\dagger}$ Corresponding Author : Hoon Jang, Department of Biomedical Science, College of Life Sciences, CHA University, Seongnam 13488, Korea. Tel: +8231-881-7248, Fax: +82-31-881-7249, E-mail: hoonjang@chamc.co.kr

This is an Open Access article distributed under the terms of the Creative Commons Attribution Non-Commercial License (http:// creative-commons.org/licenses/by-nc/3.0) which permits unrestricted non-commercial use, distribution, and reproduction in any medium, provided the original work is properly cited. 
family, and it has the ability to dimerize like other bZIP family (Akhova et al., 2005; Misra et al., 2005; Xie et al., 2008). CREBZF has been reported as a co-regulator of ATF4 and CREB3, another HCF-binding transcription factor (Misra et al., 2005; Hogan et al., 2006). Recently, CREBZF regulates bone formation and adipocyte differentiation by interacting transcription factors (Jang et al., 2014; Jang et al., 2015). Also, recent study indicated that CREBZF is induced by estrogen and closely related to implantation (Lin et al., 2013). However, this study is unclear and lacks the correlation between estrogen and CREBZF expression in endometrium of uterus. To discover the function of CREBZF during menstrual cycle, we examined the expression of CREBZF in endometrium during mouse estrous cycle.

\section{MATERIALS AND METHODS}

\section{Animals}

All animal experiments were approved by Institutional Agricultural Animal Care and Use Committee of CHA University (IACUC No. 160018). The animal studies were conducted with 3 -week old and 6 week old female ICR mice from KOATECH (Korea). Mice were housed in animal care facility at the CHA University of CHA biocomplex with a temperature-controlled environment under 12 hour dark-light cycle and free access to standard rodent diet and water. Six-weeks old female ICR mice were ovariectomized (OVX) and then following a recovery period for 2 weeks.

\section{Hormone injection}

Three-week old and OVX female ICR mice were injected subcutaneously with $17 \beta$-estradiol (E2, $10 \mathrm{ug} / \mathrm{kg}$, SigmaAldrich, USA) and Sesame oil (100 uL, Acros, USA) as control group. The Uteri were collected at $0,1,3,6,12$, and 24 hours after injection of hormones from sacrificed mice. To identify whether the expression of CREBZF is regulat- ed by E2 administration, an estrogen receptor antagonist (ICI $182,780,25 \mathrm{mg} / \mathrm{kg}$ ) was co-injected to OVX mice.

\section{Vaginal cytology}

To obtain a sample of epithelial cells from the mouse vagina some of the saline solution was flushed into mouse vagina using pipette tip to a depth of about $5 \mathrm{~mm}$. The isolated cells were placed on glass slide and dried. The cells were fixed using methanol and stained by Hematoxylin and eosin solution. The stage of the estrous cycles were determined by vaginal smear cytology (Evans \& Long, 1922).

\section{RT-PCR and real-time PCR}

To extract total RNA from four cycles of uterus and each of tissues, 6-week old female ICR mice were sacrificed and isolated after analysis of vaginal cytology. The tissues were placed into a vessel and homogenized in Trizol reagent (Invitrogen, USA) using homogenizer. The total RNA extraction was according to manufacturer's protocol. cDNA synthesis was performed using SensiFAST cDNA Synthesis Kit (Bioline, UK) according to manufacturer's protocol, and the thermal cycling conditions were as follows: reverse transcription at $45^{\circ} \mathrm{C}$ for 60 minutes, and $4^{\circ} \mathrm{C}$ for 10 minutes. Real-time PCR and RT-PCR analyses were performed in triplicate using Solg Taq DNA polymerase (Solgent, Korea) and iQ SYBR Green Supermix (Bio-Rad, USA). The thermal cycling conditions were as follows: initial denaturation at $95^{\circ} \mathrm{C}$ for 3 minutes, followed by 40 cycles of $95^{\circ} \mathrm{C}$ for 10 seconds, $60^{\circ} \mathrm{C}$ for 15 seconds, and $72^{\circ} \mathrm{C}$ for 20 seconds. Expression levels were normalized to those of endogenous glyceraldehyde 3-phosphate dehydrogenase $(G A P D H)$.

\section{Western blot analyses}

Transfected 293T cells were prepared using PRO-PREP solution (iNtRON Biotechnology, Korea) according to manufacturer's protocol. Western blot was performed using anti-CREBZF antibody (Santacruz, USA) and $\beta$-Actin 
(Santacruz, USA).

\section{Immunostaining}

Uteri isolated from mice were fixed for 1 week in $4 \%$ formaldehyde at $4^{\circ} \mathrm{C}$ for immunostaining. After fixation, uteri were embedded in paraffin and sectioned at $5 \mu \mathrm{m}$ of thickness using a microtome and put on microscope slides (HistoBond, Germany). Sections were deparaffinized as follows; slides were dipped three times for $5 \mathrm{~min}$ in xylene (Biosesang, Korea), two times for $5 \mathrm{~min}$ in 95\% ethanol, one time for $5 \mathrm{~min}$ in $90 \%, 80 \%, 70 \%, 50 \%$ ethanol, and $10 \mathrm{~min}$ in distilled water. For immunofluorescence, deparaffinized slides were placed into a Retrieval Steamer (IHCworld, USA) and antigen retrieval was performed using an Epitope Retrieval Solution (IHCworld, USA) according to the manufacturer's protocol. After washing in PBS, excess PBS was removed, and blocking buffer (4\% BSA and 5\% rabbit serum in PBS) was added to the slides. The slides were incubated in a humidified chamber for $4 \mathrm{~h}$ at room temperature (RT). Then, the slides were incubated with the following primary antibodies for $16 \mathrm{~h}$ at $4^{\circ} \mathrm{C}$ : Goat polyclonal antibody against CREBZF (1:1000, Santacruz, USA), rabbit polyclonal antibody against PR (1:500, Santacruz, USA). After three washes in PBS, the slides were incubated with Alexa 488 mouse anti-Goat and Alexa 546 mouse anti-rabbit (1:1,500, Invitrogen, UK) for $2 \mathrm{~h}$ at RT. A 4',6-diamidino-2-phenylindole (DAPI, 1:20000, Life Technologies, USA) was used to stain the nuclei. Mounting medium (DAKO, USA) was applied to the tissue sections prior to covering them with glass coverslips. All images were obtained using a confocal microscope (Leica) and analyzed by the imaging software LAS lite (Leica). For immunohistochemistry, antigen-retrieved sections were washed with PBS with $0.5 \%$ Tween 20 (PBS/T) and incubated in $0.5 \%$ hydrogen peroxide in distilled water for 10 minutes (min) to quench endogenous peroxidase activity. Then, the slides were washed two times in cold PBS/T for 10 min. Blocking was conducted using blocking solution
(4\% BSA and 5\% rabbit serum in PBS/T) for $2 \mathrm{~h}$ at RT. The sections were incubated in the previously described primary antibody against CREBZF $(1: 1,500)$ for $16 \mathrm{~h}$ at $4{ }^{\circ} \mathrm{C}$, followed by incubation in diluted rabbit HRP-conjugated anti-goat antibody (1:500, Invitrogen, UK) for $1 \mathrm{~h}$ at RT. The slides were washed in PBS/T three times for $5 \mathrm{~min}$ and put into distilled water. A 3,3'-diaminobenzidine (DAB) solution (Vector Laboratories, USA) was dropped on the sections until the desired staining was obtained, according to the manufacturer's manual. The sections were counterstained with hematoxylin and dehydrated using ethanol and xylene. The mounting was performed using Permount mounting medium (Fisher Scientific, USA), and then coverslips were added.

\section{Statistical analysis}

All values are reported as standard error of mean. The results were analyzed using student's $t$-test and one-way ANOVA for statistical evaluation. For all analyses, $p<0.05$ was considered to be statistically significant.

\section{RESULTS AND DISCUSSION}

\section{CREBZF expression is significantly increased in} the uterus

To examine expression levels of $C R E B Z F$ transcript in various tissues, we performed RT-PCR and qRT-PCR using respective cDNA from 6-weeks old mice organs and designed mouse $C R E B Z F$ primers (Table 1). The CREBZF transcript was detected in various tissues and was highly expressed in uterus than other tissues in adult mice (Fig. 1A-B). Recent studies showed that CREBZF expression is regulated by insulin and regulates hepatic gluconeogenesis (Kim \& Ahn, 2016; Lee et al., 2016). These results suggest that CREBZF plays major roles in liver tissue and that expression is also high in liver tissue. Therefore, we examined the expression of CREBZF in several tissues of mice based on liver tissue, and found that CREBZF expression 
Table 1. Primer sequences

\begin{tabular}{|c|c|}
\hline Genes & Primer sequences \\
\hline \multirow{2}{*}{$\begin{array}{l}\text { Human } \\
\text { GAPDH }\end{array}$} & F 3- AAGGTGAAGGTCGGAGTCAAC - 5 \\
\hline & R 3'- GGGGTCATTGATGGCAACAAT - 5 \\
\hline \multirow{2}{*}{$\begin{array}{c}\text { Human } \\
\text { CREBZF }\end{array}$} & F 3-ATGAGGCATAGCCTGACCAAG - 5 \\
\hline & R 3- GCAGCGAACAAGTTGCAGC - 5 \\
\hline \multirow{2}{*}{$\begin{array}{c}\text { Mouse } \\
\text { GAPDH }\end{array}$} & F 3'- AGGTCGGTGTGAACGGATTTG - 5' \\
\hline & R 3- TGTAGACCATGTAGTTGAGGT - 5 \\
\hline \multirow{2}{*}{$\begin{array}{c}\text { Mouse } \\
\text { CREBZF }\end{array}$} & F 3'- CTGCCCGTCTTAATCGGCTC - 5 \\
\hline & R 3- CCGTAGGTAGCGACTCTCCTC - 5 \\
\hline
\end{tabular}

GAPDH, Glyceraldehyde 3-phosphate dehydrogenase; CREBZF, cyclic AMP-response element binding protein zhangfei.

is highest in the uterus. Taken together, our results indicated that CREBZF has an important role in uterus.

\section{CREBZF is highly expressed at estrus cycle and} localized in epithelial cells of endometrium

To evaluate expression patterns of $C R E B Z F$ in the uterus, we identified changes in expression over the development of the estrous cycle such as diestrus, proestus, estrus, and metestrus. Semi-quantitative PCR showed that $C R E B Z F$ was significantly increased at estrus stage (Fig. 2A). Because the estrogen is dramatically decreased at estrus stage (Miller \& Takahashi, 2014), it suggested that CREBZF is sensitively regulated by estrogen. To obtain definite results, we performed an antibody test by western blot using 293 T cells transfected with the mouse CREBZF overexpression vector and found that the antibody was well working (Fig. 2B). To evaluate the localization of CREBZF protein during estrous cycle, we conducted immunohistochemistry analysis in uterine sections collected from all stages of the estrous cycle using CREBZF antibody. CREBZF expression was more highly expressed in the LE and GE than stroma cells in the endometrium of uterus at

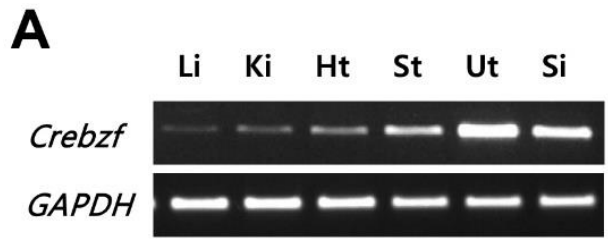

B

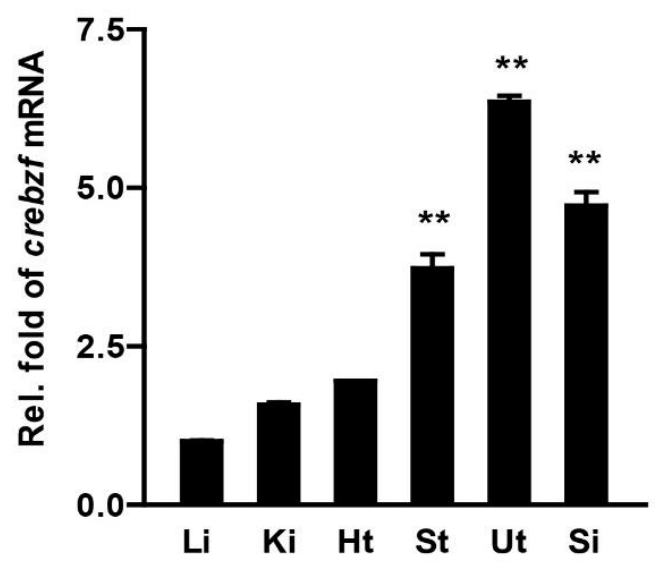

Fig. 1. CREBZF expression in mouse tissues. (A) RTPCR and (B) qRT-PCR analyses of CREBZF transcript expression were performed using total RNA from 6-weeks-old mouse tissues. Mouse GAPDH was used as an internal control. Expression levels were calculated from $\Delta \Delta \mathrm{CT}$ values and normalized against GAPDH mRNA. The fold changes were evaluated by comparing the level of $C R E B Z F$ mRNA at Liver. $* *, p$-value $(p<0.01)$. CREBZF, cyclic AMP-response element binding protein zhangfei; Li, Liver; Ki, Kidney; Ht, Heart; St, Stomach; Ut, Uterus; Si, Small intestine; PCR, polymerase chain reaction.

the estrus stage (Fig. 2C left panel). Each stage of the estrous cycle was identified by the ratio of leukocytes and epithelial cells in the vagina using hematoxylin and eosin staining method (Fig. 2C right panel). In the uterus, the endometrium is dynamically regulated throughout the estrous cycle and it consists of the LE, GE, and the stroma. The proliferation and differentiation of endometrium were closely controlled by two major ovarian hormones, E2 and P4. The sexual steroid hormones from ovary controlled estrous cycle which is critical for a pregnancy due to mod- 


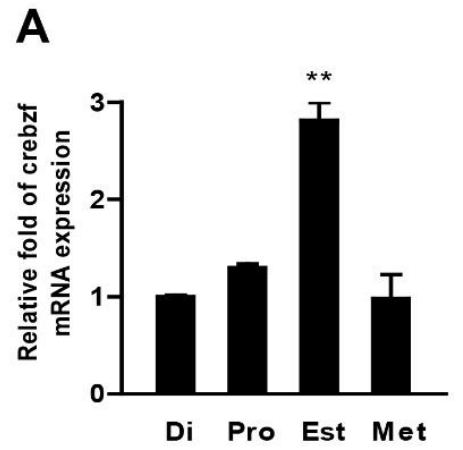

B
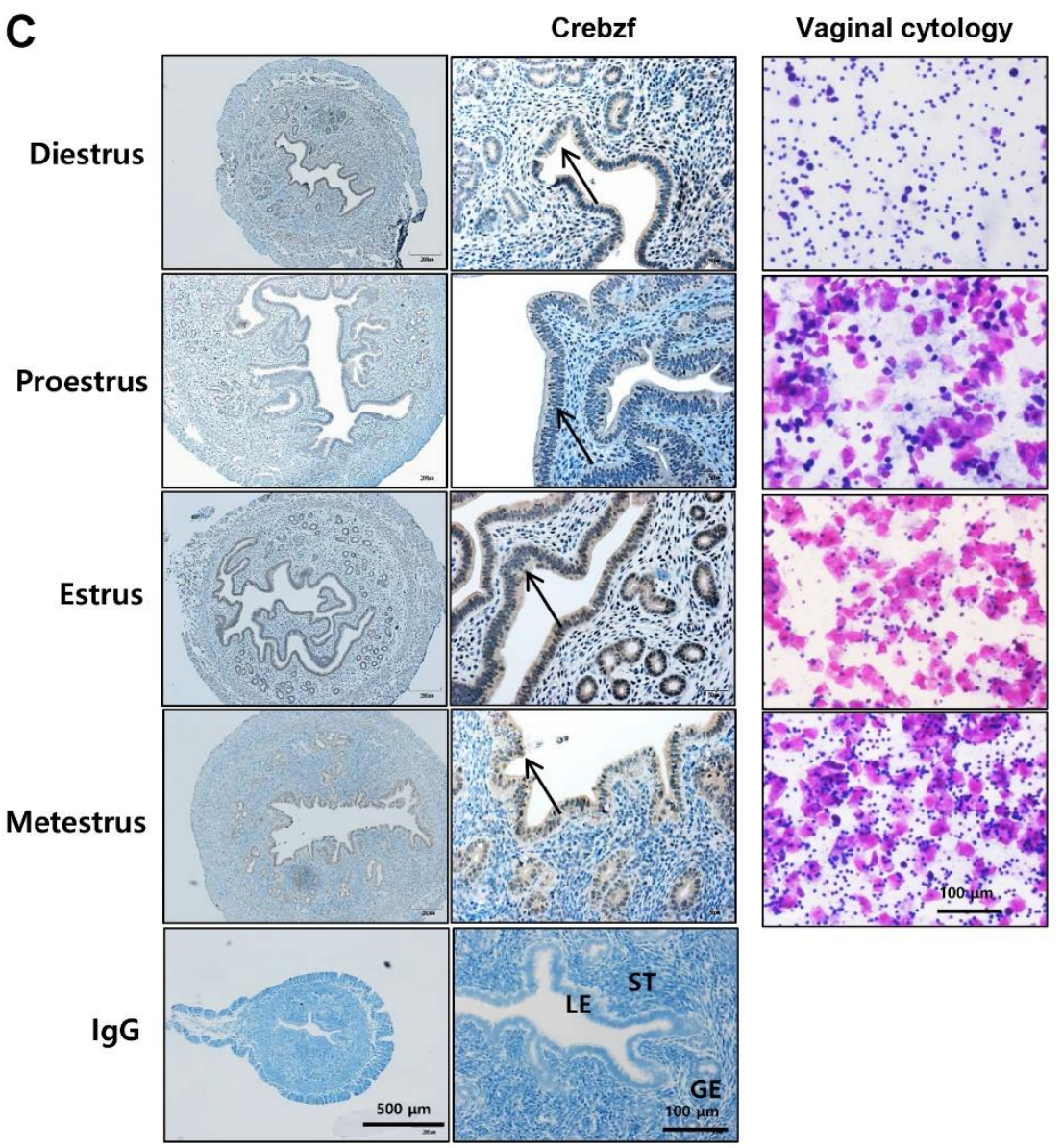

Fig. 2. CREBZF expression in mouse uterus during the estrous cycle. A: qRT-PCR analysis of $C R E B Z F$ transcript expression in mouse uteri at different stages in the estrous cycle. Expression levels were calculated from $\triangle \triangle C T$ values and normalized against GAPDH mRNA. The fold changes were evaluated by comparing the level of CREBZF mRNA at diestrus B: Antibody test of CREBZF for the experiment. Mouse CREBZF gene was cloned into pcDNA3.1 expression vector, and then transfected to hek293T cells with blank vector for $48 \mathrm{~h}$. Western blot analysis showed that anti-CREBZF antibody detected the protein product of cloned CREBZF. (C, left panel) Immunohistochemistry of CREBZF at different stages of the estrous cycle in mouse uterus. Sections of uteri were stained with goat polyclonal antibody against CREBZF. Black arrow indicates LE. (C, right panel) Representative images of vaginal smear analysis conforming stages of the estrous cycle. Pink stained cells are cornified epithelial cells and purple dots are leukocyte. The scale bars represented into figures. CREBZF, cyclic AMP-response element binding protein zhangfei; LE, Luminal epithelium; GE, glandular epithelium; ST, Stroma; PCR, polymerase chain reaction. 
ulate uterus. Circulating E2 levels are increased at the proestrus stage and then decline during estrus stage (Walmer et al., 1992; McLean et al., 2012), whereas P4 begin to elevate after estrus and peak at diestrus (McLean et al., 2012; Miller \& Takahashi, 2014). Also, E2 stimulates uterine cell proliferation and $\mathrm{P} 4$ stimulates uterine cell differentiation (Finn \& Martin, 1974). In addition, numerous studies demonstrated that E2 and P4 regulates various signaling factors (Altmäe et al., 2010; Cha et al., 2012; Choi et al., 2016; Rosario et al., 2016). Taken together, our results suggest that CREBZF expression is closely regulated by blood E2 levels in epithelial cells of endometrium.

\section{CREBZF expression is suppressed by estradiol} in epithelium of pre-pubertal mice

To investigate whether CREBZF is regulated by E2 treatment in the uterus of prepubertal mice, we performed E2 injection to 3-week-old female ICR mice. Semiquantitative PCR and immunohistochemistry were performed using collected uterus at various time points. The results showed that E2 treatment dramatically reduced CREBZF mRNA expression from 3 hours after administration (Fig. 3A). CREBZF protein expression was specifically declined by E2 in only epithelium of endometrium (Fig. 3B). To further confirm that CREBZF is downregulated by E2 in other cells, E2 was treated to Ishikawa cells, a human endometrial cell line. Similar to the previous results, E2 reduced human CREBZF mRNA expression level (Fig. 3C). Next, to further determine whether CREBZF was reduced by E2 in OVX mice, we treated the E2 to OVX mice and collected the uteri at various times. A progesterone receptor (PR), an intra-uterine E2 marker, was also identified to conform whether E2 was administrated correctly. Immunofluorescence showed that expression of CREBZF was decreased in the OVX mouse endometrium while it was expressed only in epithelium, but the expression of PR markedly decreased in epithelium and dramatically increased in stroma after 12 hours of
E2 injection (Fig. 4). Previous studies have shown that the E2 response has progressed from about 2 hours after E2 injection (Seong et al., 2014). In light of that study, it is possible to interpret the result of qRT-PCR that did not affect the expression of CREBZF in uterine samples after 1 hour of E2 injection (Fig. 3A). In addition, since the reaction by $\mathrm{E} 2$ is rapid in cell based experiments, the expression level of CREBZF is decreased even at 1 hour (Fig. 3C). Interestingly, our results indicate that CREBZF is expressed specifically in endometrial epithelium, whereas PR is expressed in the epithelium when the E2 concentration is low, but is specifically expressed in the stroma when the E2 concentration is high Respectively (Fig. 4). These results indicate that the expression of PR is rapidly decreased when blood E2 level is elevated, thereby controlling the environment of endometrial epithelium. The CREBZF has been reported as a co-regulator without a DNA binding domain and plays a role through interactions with major regulators to perform most roles (Misra et al., 2005; Hogan et al., 2006; Jang et al., 2015). Recently, Seo et al have suggested the possibility of role of the transcription factor egrl, an important regulator of cell growth, differentiation, and apoptosis, in the uterus (Seo et al., 2014). These results suggest that CREBZF may interact with critical factors as egrl or PR.

\section{Estrogen-dependent expression of CREBZF is} mediated through estrogen receptors

In order to examine whether CREBZF expression is affected by E2 in estrogen receptor dependent (ER) manner, OVX ICR mice were co-treated with an ER antagonist, ICI 182,780 (ICI), and E2. As shown in Fig. 5, immunohistochemistry presented that ICI treatment significantly restored E2-induced CREBZF suppression. Most induction of target factors by hormones occurs via hormone receptor, stimulated by extra nuclear actions. We demonstrated that the CREBZF expression is regulated by the ER signaling pathway through experiment of co-administration of ICI. 

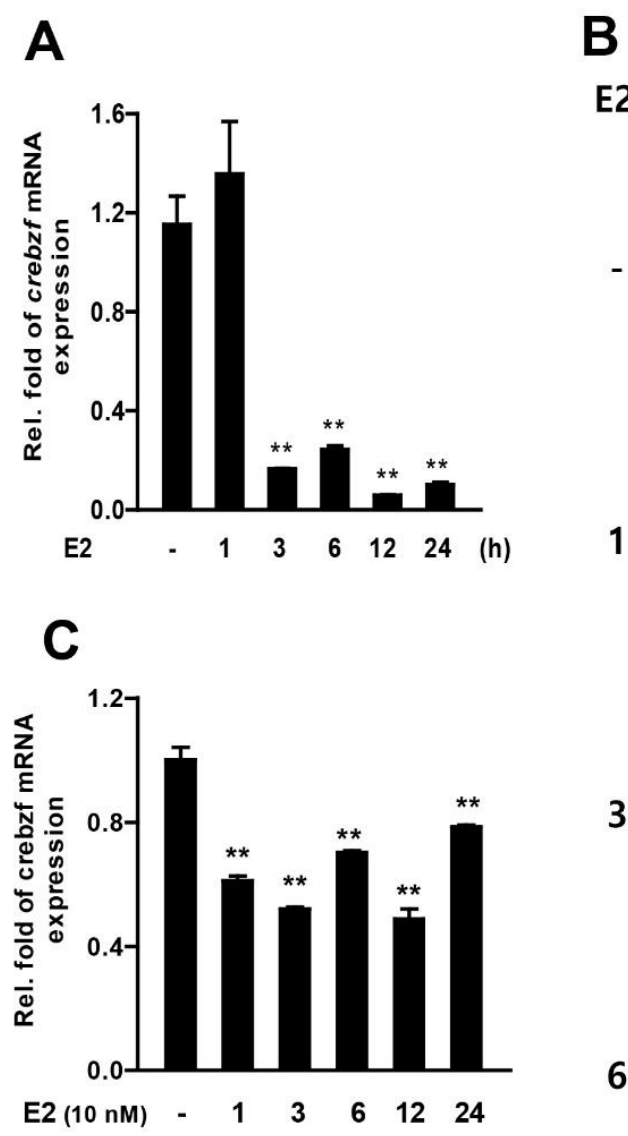

E2
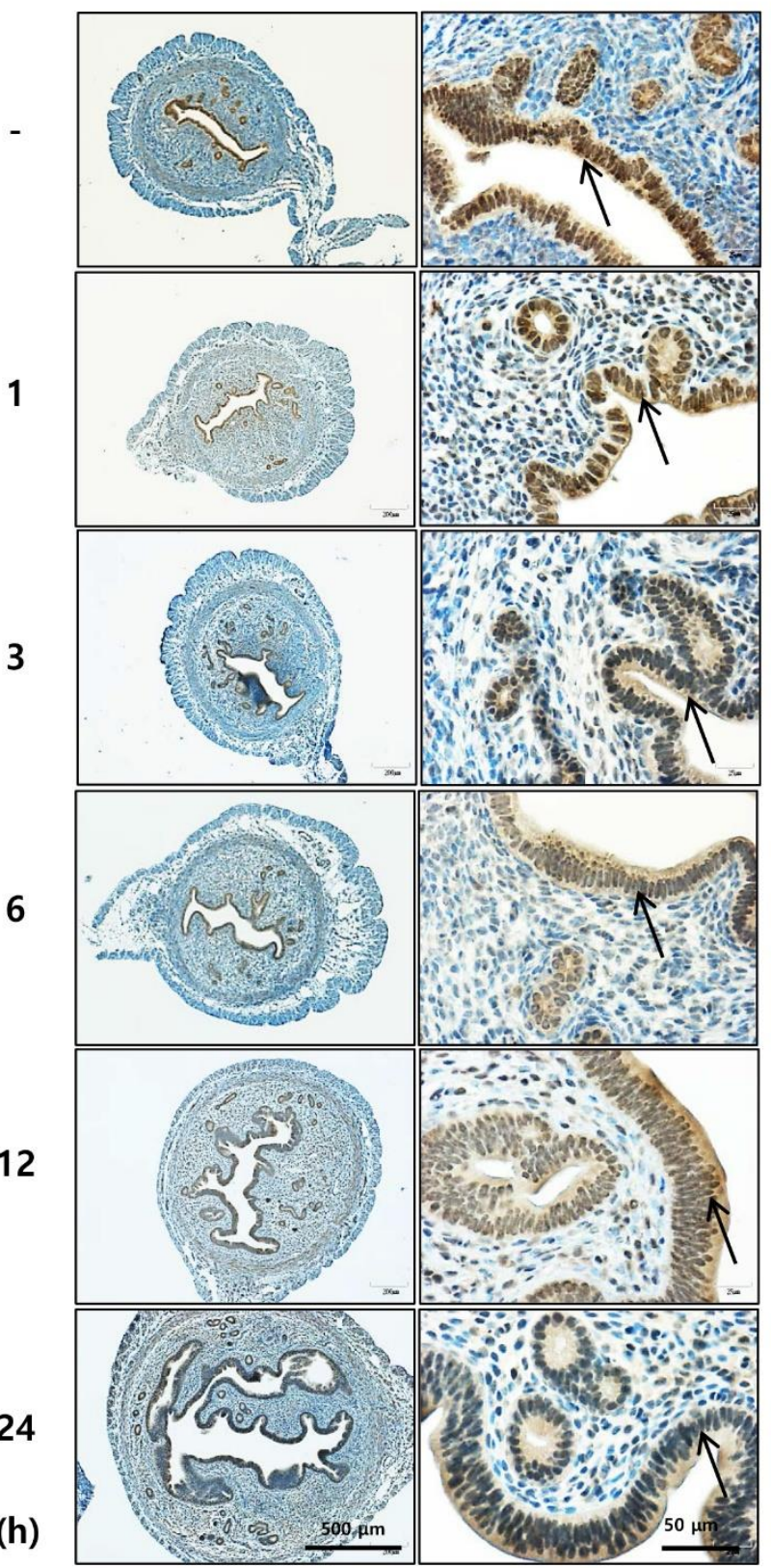

Fig. 3. Regulation of CREBZF expression by $\mathbf{E} 2$ in the uterus of pre-pubertal mouse. A: qRT-PCR analysis for examining relative fold of $C R E B Z F \mathrm{mRNA}$ in the uteri of 3-week-old mice after E2 (10 ug/ $\mathrm{kg}$ ) or sesame oil injection. GAPDH was used for normalization. B: Immunohistochemistry to localize and identify CREBZF protein expression in the endometrium. Sections of uteri were stained with goat polyclonal antibody against CREBZF. C: IgG is without anti-CREBZF antibody for negative control. qRT-PCR analysis of human CREBZF mRNA expression level in the human endometrial stroma (Ishikawa) cells after E2 treatment for different time point. Expression levels were calculated from $\triangle \Delta \mathrm{CT}$ values and normalized against GAPDH mRNA. The fold changes were evaluated by comparing the level of $C R E B Z F$ mRNA at Liver. $* *, p$-value $(p<0.01)$. CREBZF, cyclic AMP-response element binding protein zhangfei; PCR, polymerase chain reaction. 
E2

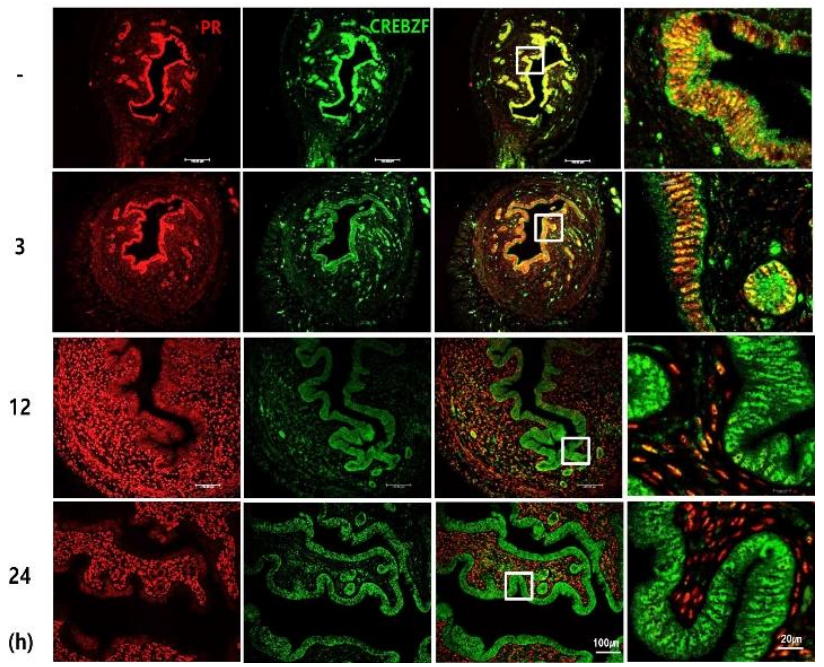

Fig. 4. Localization of CREBZF and PR after E2 injection in the endometrium of OVX mice. Confocal microscopic images showed the localization of CREBZF and PR in uteri of ovariectomized mice after treated with E2. Sections of uteri were stained with antibody against progesterone receptor (PR, red) and CREBZF (green). The enlarged images corresponding to the box lined in white on merged images. The scale bars represented into figures. OVX, ovariectomized.

Further study will be conducted to determine what mechanism is involved in CREBZF among various ER signaling pathways in endometrium.

In conclusion, we showed that CREBZF was specifically expressed in the uterine epithelium and its expression was regulated by E2 levels, and also, the CREBZF expression is moderated by ER signaling pathway. It will give us better understanding the function and mechanism of CREBZF in dynamic cycles of uterus.

\section{ACKNOWLEDGEMENTS}

This research was supported by a grant from Basic Science Research Program through the National Research Foundation of Korea (NRF) funded by the Ministry of Education (2016R1D1A1B03931983).
Oil

E2 $E 2+\mathrm{ICI} 182,780$

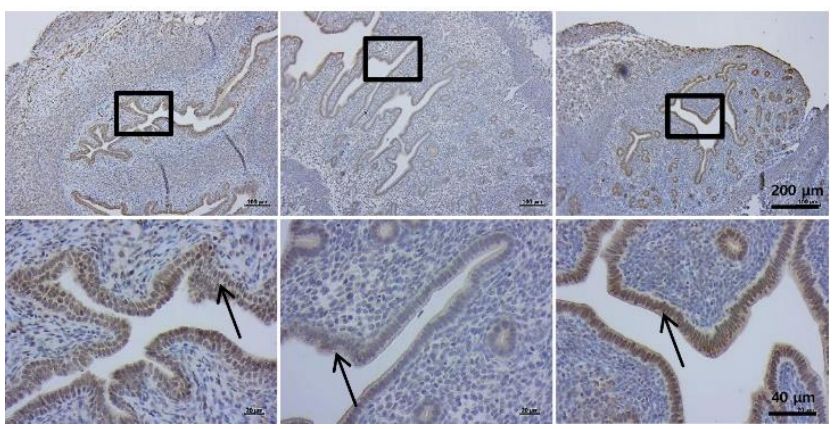

Fig. 5. Regulation of CREBZF expression through estrogen receptor (ER) signaling pathway. The OVX mice were treated with ICI 182,780 (ICI), an ER antagonist, and E2 (10 ug/ $\mathrm{kg})$. The expression pattern of CREBZF in epithelium of endometrium was examined by immunohistochemistry analysis using anti-CREBZF antibody. The enlarged images corresponding to the box lined in black and black arrows indicate luminal epithelium. The scale bars represented into figures. OVX, ovariectomized.

\section{REFERENCES}

Akhova O, Bainbridge M, Misra V (2005) The neuronal host cell factor-binding protein Zhangfei inhibits herpes simplex virus replication. J Virol 79:14708-14718.

Altmäe S, Martinez-Conejero JA, Salumets A, Simon C, Horcajadas JA, Stavreus-Evers A (2010) Endometrial gene expression analysis at the time of embryo implantation in women with unexplained infertility. Mol Hum Reprod 16:178-187.

Calle EE, Kaaks R (2004) Overweight, obesity and cancer: Epidemiological evidence and proposed mechanisms. Nat Rev Cancer 4:579-591.

Cha J, Sun X, Dey SK (2012) Mechanisms of implantation: Strategies for successful pregnancy. Nat Med 18:17541767.

Choi Y, Kim HR, Lim EJ, Park M, Yoon JA, Kim YS, Kim EK, Shin JE, Kim JH, Kwon H, Song H, Choi DH (2016) Integrative analyses of uterine transcriptome and MicroRNAome reveal compromised LIF-STAT3 sig- 
Regulation of Cyclic AMP-Response Element Binding Protein Zhangfei (CREBZF) Expression by Estrogen in Mouse Uterus

naling and progesterone response in the endometrium of patients with recurrent/repeated implantation failure (RIF). PloS one 11:e0157696.

Evans HM, Long JA (1922) Characteristic effects upon growth, oestrus and ovulation induced by the intraperitoneal administration of fresh anterior hypophyseal substance. Proc Natl Acad Sci USA 8:38-39.

Finn CA, Martin L (1974) The control of implantation. J Reprod Fertil 39:195-206.

Gruber CJ, Tschugguel W, Schneeberger C, Huber JC (2002) Production and actions of estrogens. N Engl J Med 346:340-352.

Hewitt SC, Harrell JC, Korach KS (2005) Lessons in estrogen biology from knockout and transgenic animals. Annu Rev Physiol 67:285-308.

Hogan MR, Cockram GP, Lu R (2006) Cooperative interaction of Zhangfei and ATF4 in transactivation of the cyclic AMP response element. FEBS Letters 580:5862.

Hu J, Gray CA, Spencer TE (2004) Gene expression profiling of neonatal mouse uterine development. Biol Reprod 70:1870-1876.

Huang CC, Orvis GD, Wang Y, Behringer RR (2012) Stromal-to-epithelial transition during postpartum endometrial regeneration. $\mathrm{PloS}$ one $7: \mathrm{e} 44285$.

Jang H, Kim EJ, Park JK, Kim DE, Kim HJ, Sun WS, Hwang S, Oh KB, Koh JT, Jang WG, Lee JW (2014) SMILE inhibits BMP-2-induced expression of osteocalcin by suppressing the activity of the RUNX2 transcription factor in MC3T3E1 cells. Bone 61:10-18.

Jang H, Kim HJ, Kim DH, Park JK, Sun WS, Hwang S, Oh KB, Jang WG, Lee JW (2015) Small heterodimer partner-interacting leucine zipper protein inhibits adipogenesis by regulating peroxisome proliferator-activated receptor $\gamma$ activity. Life Sci 132:49-54.

Kim MY, Ahn YH (2016) SMILE is an insulin-inducible transcriptional corepressor of hepatic gluconeogenic gene programs. Diabetes 65:14-15.
Lee JM, Seo WY, Han HS, Oh KJ, Lee YS, Kim DK, Choi $\mathrm{S}$, Choi BH, Harris RA, Lee CH, Koo SH, Choi HS (2016) Insulin-inducible SMILE inhibits hepatic gluconeogenesis. Diabetes 65:62-73.

Lin P, Chen F, Wang N, Wang X, Li X, Zhou J, Jin Y, Wang A (2013) CREBZF expression and hormonal regulation in the mouse uterus. Reprod Biol Endocrinol 11:110.

Martín J, Domínguez F, Ávila S, Castrillo JL, Remohí J, Pellicer A, Simón C (2002) Human endometrial receptivity: Gene regulation. J Reprod Immunol 55:131-139.

McLean AC, Valenzuela N, Fai S, Bennett SA (2012) Performing vaginal lavage, crystal violet staining, and vaginal cytological evaluation for mouse estrous cycle staging identification. J Vis Exp e4389.

Miller BH, Takahashi JS (2014) Central circadian control of female reproductive function. Front Endocrinol 4:195.

Misra V, Rapin N, Akhova O, Bainbridge M, Korchinski P (2005) Zhangfei is a potent and specific inhibitor of the host cell factor-binding transcription factor Luman. J Biol Chem 280:15257-15266.

Riesewijk A, Martín J, van Os R, Horcajadas JA, Polman J, Pellicer A, Mosselman S, Simón C (2003) Gene expression profiling of human endometrial receptivity on days $\mathrm{LH}+2$ versus $\mathrm{LH}+7$ by microarray technology. Mol Hum Reprod 9:253-264.

Rosario GX, Cheng JG, Stewart CL (2016) Gene expression analysis in the compartments of the murine uterus. Differentiation 91:42-49.

Seo BJ, Son JW, Kim HR, Hong SH, Song H (2014) Identification of egrl direct target genes in the uterus by in silico analyses with expression profiles from mRNA microarray data. Dev Reprod 18:1-11.

Seong Y, Moon J, Kim J (2014) Egrl mediated the neuronal differentiation induced by extremely low-frequency electromagnetic fields. Life Sci 102:16-27.

Walmer DK, Wrona MA, Hughes CL, Nelson KG (1992) Lactoferrin expression in the mouse reproductive tract 
during the natural estrous cycle: Correlation with circulating estradiol and progesterone. Endocrinology 131:1458-1466.

Xie YB, Lee OH, Nedumaran B, Seong HA, Lee KM, Ha
H, Lee IK, Yun Y, Choi HS (2008) SMILE, a new orphan nuclear receptor SHP-interacting protein, regulates SHP-repressed estrogen receptor transactivation. Biochem J 416:463-473. 